\title{
Double-mutant myeloproliferative neoplasms
}

\author{
Stephen E. Langabeer ${ }^{1}$
}

Received: 21 August 2018 / Accepted: 28 August 2018 / Published online: 29 August 2018

o) Springer Science+Business Media, LLC, part of Springer Nature 2018

To the editor,

In the recent review of myeloproliferative neoplasms (MPN), Helbig summarizes the current evidence of how the three major types of MPN driver mutations (JAK2 V617F, $C A L R$ exon 9 and $M P L$ exon 10) influence disease phenotype and impact on prognosis. In this review, it is stated that these three mutation types are mutually exclusive [1]. However, in addition to an increased annotation of cases in which an MPN harbouring one of these types of mutations co-exists with $B C R$ - $A B L 1$-positive chronic myeloid leukemia [2], there is a growing awareness that a combination of any two of these types of mutations can occur concurrently in a minor, but significant proportion of patients with the classical MPN of polycythemia vera, essential thrombocythemia and primary myelofibrosis. In one of the largest series reported to date, double-mutant MPN appear to possess specific presenting features, dependent on which of the two mutations are present [3].

Coincident with this increasing appreciation of the double-mutant MPN entity is the consequence for MPN molecular diagnostics. Step-wise algorithms for the JAK2 V617F, CALR and MPL mutations in which once a mutation is detected no further analysis is performed must now be reconsidered. Simultaneous identification of the three driver mutations by targeted next-generation sequencing approaches would largely overcome this issue and would likely enhance identification of further cases, necessary for stratified risk-assessment and selection of treatment.

\section{Compliance with ethical standards}

Conflict of interest The author declares that there is no conflict of interest.

\section{References}

1. Helbig G. Classical Philadelphia-negative myeloproliferative neoplasms: focus on mutations and JAK2 inhibitors. Med Oncol. 2018;35:119.

2. Boddu P, Chihara D, Masarova L, Pemmaraju N, Patel KP, Verstovsek S. The co-occurrence of driver mutations in chronic myeloproliferative neoplasms. Ann Hematol. 2018. https://doi. org/10.1007/s00277-018-3402-x.

3. Mansier O, Luque Paz D, Ianotto JC, et al. Clinical and biological characterization of MPN patients harbouring two driver mutations, a French intergroup of myeloproliferative neoplasms (FIM) study. Am J Hematol. 2018;93:E84-6.
Stephen E. Langabeer

slangabeer@stjames.ie

1 Cancer Molecular Diagnostics, Central Pathology

Laboratory, St. James's Hospital, Dublin D08 E9P6, Ireland 